\title{
An Odd Case of Melena: Chronic Celiac Artery Dissection and Hepatic Arterioduodenal Fistula
}

\author{
Lijesh Kumar ${ }^{1}$ Cyriac Abby Philips ${ }^{2} \quad$ Philip Augustine ${ }^{3}$ \\ ${ }^{1}$ Department of Interventional Radiology, PVS Institute of Digestive \\ Diseases, PVS Memorial Hospital, Cochin, Kerala, India \\ 2Department of Hepatology and Liver Transplant Medicine, PVS \\ Institute of Digestive Diseases, PVS Memorial Hospital, Cochin, \\ Kerala, India \\ ${ }^{3}$ Department of Gastroenterology, PVS Institute of Digestive \\ Diseases, PVS Memorial Hospital, Cochin, Kerala, India \\ J Gastrointestinal Abdominal Radiol ISGAR 2018;1:72-74
}

\begin{abstract}
Address for correspondence Lijesh Kumar, MD, PVS Memorial Hospital Pvt. Ltd., Ernakulam 682017 Kerala, India

(e-mail: lijeshkumar45@gmail.com).
\end{abstract}

\author{
Abstract \\ Keywords \\ - dissecting aneurysm \\ - computed \\ tomography \\ - melena \\ - hepatoduodenal \\ fistula \\ - pseudoaneurysm \\ - common hepatic \\ artery
}

We present the case of a 54-year-old chronic alcoholic man who presented to the emergency department with intermittent melena for 3 days. Imaging revealed a large dissecting pseudoaneurysm of the common hepatic artery with an arterioduodenal fistula in the second part of the duodenum. The pseudoaneurysm and fistula were managed with refined interventional techniques, and supply to the hepatic arteries was salvaged. In cases of melena that are difficult to diagnose through traditional means, early imaging and interventional management can prove beneficial and improve short-term survival.

\section{Introduction}

A true aneurysm is saccular or a fusiform dilatation of an artery with a proper arterial wall. A pseudoaneurysm, by contrast, is a contained hematoma resulting from a defect in the wall of an artery, with the wall formed by surrounding structures. The most common site of hepatic artery aneurysms is the common hepatic artery (CHA). Atherosclerosis and other congenital and degenerative processes are the common causes of hepatic artery aneurysms. Abdominal trauma, percutaneous interventional techniques, transarterial chemoembolization of liver tumors, vascular surgery, and liver transplantation are the reasons for the increasing occurrence of hepatic pseudoaneurysms. The most common presentation of hepatic artery pseudoaneurysm is rupture into the peritoneal cavity with less frequent presentations of rupture into the gastrointestinal tract (mainly the duodenum) and, very rarely, into the common bile duct. The clinical picture varies from acute upper gastrointestinal (GI) bleeding and shock to smaller herald bleeds. ${ }^{1}$ Here, we report the case of an acute upper GI hemorrhage manifesting as melena, caused by a dissecting aneurysm of the common hepatic artery, in the absence of abdominal trauma, with formation of a hepatic arterioduodenal fistula.

\section{Case Report}

A 54-year-old chronic alcoholic male and binge drinker, without any known chronic illnesses, metabolic syndrome, or past abdominal surgeries or trauma, presented to the emergency department with a 1-week history of intermittent melena, associated with postural symptoms for 1 day. Clinical examination was significant for hypotension (mean arterial pressure $60 \mathrm{~mm} \mathrm{Hg}$ ), tachycardia and pallor with diaphoresis without significant abdominal signs. Blood investigations showed anemia (hemoglobin $8.6 \mathrm{~g} / \mathrm{L}$, normal 13-16 g/L), increased lactate $(5.6 \mathrm{mmol} / \mathrm{L}$, normal $<1 \mathrm{mmol} / \mathrm{L})$, and preserved renal and liver function tests. Upper GI endoscopy revealed an ulcerated mass in the second part of the duodenum with an inferior opening separate from the mass. A computed received

September 8, 2018 accepted after revision

October 5, 2018
License terms

()(1) $\Theta \circledast$ 
tomographic (CT) angiography of the abdomen revealed a large dissecting aneurysm of the common hepatic artery with a thrombosed false lumen ( $\boldsymbol{- \text { Fig. }} \mathbf{1 \mathrm { A }}$, arrow) compressing and displacing the pyloric region. A fistula was noted between the thrombosed false lumen and second part of the duodenum $(-$ Fig. 1B, arrow). A tight stenosis ( - Fig. 1C, thick arrow) was noted at the ostium of the common hepatic artery, with the dissecting aneurysm ( - Fig. $1 \mathrm{C}$, thin arrow) extending distally up to just proximal to the hepatic artery bifurcation. The gastroduodenal artery was not visualized, with tiny inferior pancreaticoduodenal branches noted supplying the hepatic artery near the bifurcation. A multidisciplinary team recommended management via interventional radiology techniques. A conventional celiac axis angiogram via a $5 \mathrm{~F}$ Simmons catheter revealed a $4.2 \times 2.9-\mathrm{cm}$ pseudoaneurysm arising from the CHA ( - Fig. 2A, arrow). The CHA was cannulated with a $2.8 \mathrm{~F}$ Progreat microcatheter (Terumo; Tokyo, Japan), and the distal end of the aneurysm was embolized with two interlock coils (Boston Scientific, Marlborough, MA) and two pushable coils (Cook Medical, Bloonington, IN) ( - Fig. 2B, thick arrow). Thereafter, a $14-\mathrm{mm} \times 50-\mathrm{cm}$ interlock coil was deployed into the aneurysmal sac. The microcatheter was gently pulled back into the stenosed CHA ostia, and stability was assessed. This was followed by an injection of glue and Lipiodol mixture (1:1) to seal the ostia ( - Fig. 2B, thin arrow). Celiac axis angiogram showed no filling of the pseudoaneurysm ( - Fig. 2C). On follow-up imaging after 14 days, complete obliteration persisted and the hepatic artery branches were seen filling via inferior pancreaticoduodenal arcade ( $\bullet$ Fig. 1D, arrows). The patient remained well.

\section{Discussion}

Aneurysms of the celiac axis (4\% of visceral aneurysm) are rare and mostly associated with congenital diseases, atherosclerosis, fibromuscular dysplasia, vasculitis, trauma, or segmental arterial mediolysis-a nonarteriosclerotic, noninflammatory arteriopathy, most commonly found in abdominal arteries. Spontaneous dissection, most often seen with the superior mesenteric artery of the celiac trunk, is uncommon and presents clinically with acute or chronic abdominal pain. $^{2}$ Hepatic artery aneurysmal dissection presents mostly as deranged liver function or hemoperitoneum with hypovolemic shock. Involvement of the hepatic artery in the form of pseudoaneurysm secondary to isolated dissection of the common hepatic artery is still rarer. Hepatic artery pseudoaneurysms commonly occur secondary to arterial trauma, intra-abdominal or retroperitoneal inflammation
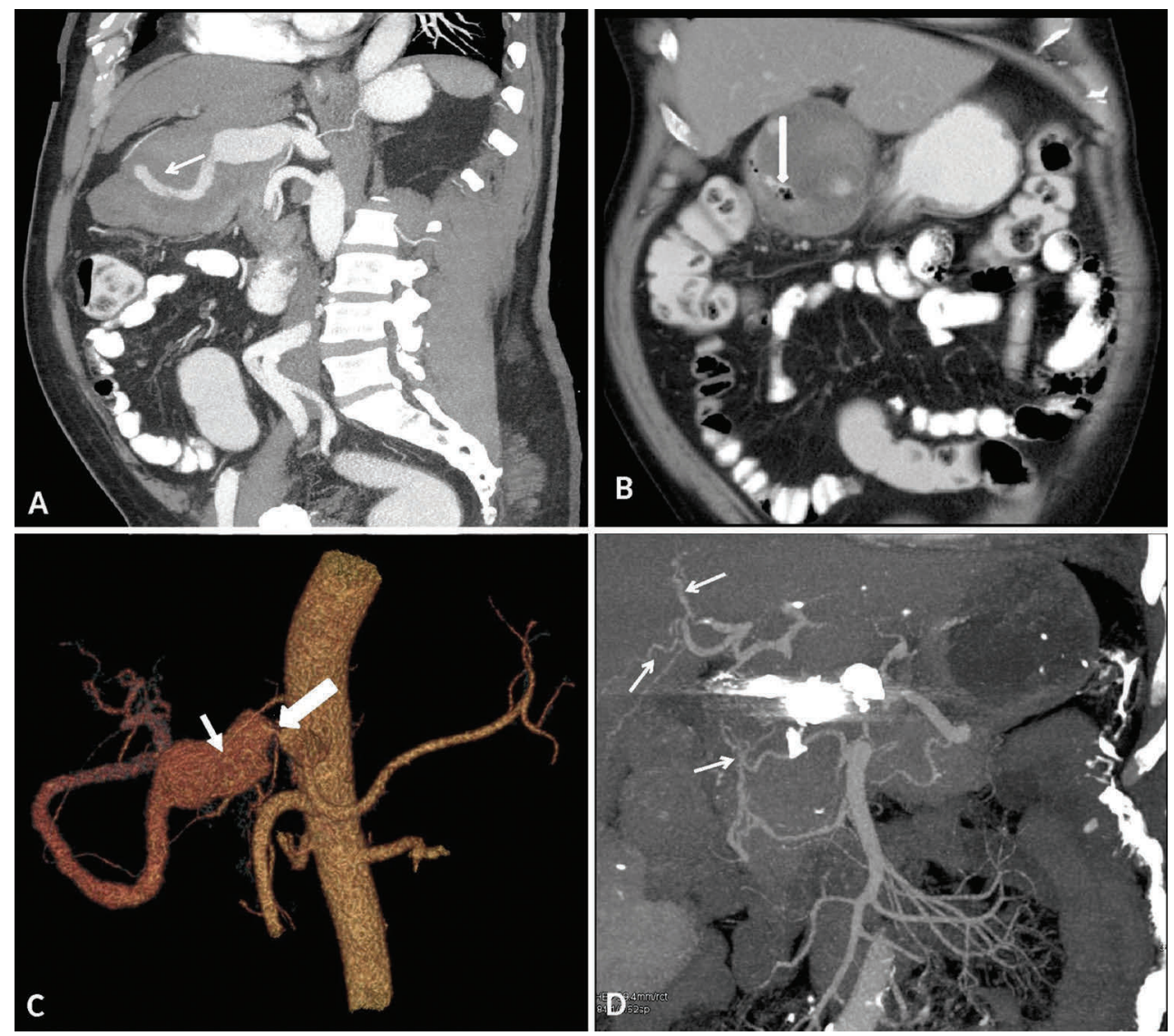

Fig. 1 CT angiography of the abdomen showing a large dissecting aneurysm of the common hepatic artery with thrombosed false lumen (A, arrow) compressing and displacing the pyloric region, and communicating with the second part of the duodenum (B, arrow) associated with a tight stenosis of $\mathrm{CHA}$ ostia (C, thick arrow) with the dissecting aneurysm extending distally up to the hepatic arterial bifurcation (C, open arrow); postinterventional treatment follow-up imaging showing complete obliteration of aneurysm and intrahepatic arterial collateral circulation (D, arrows). 

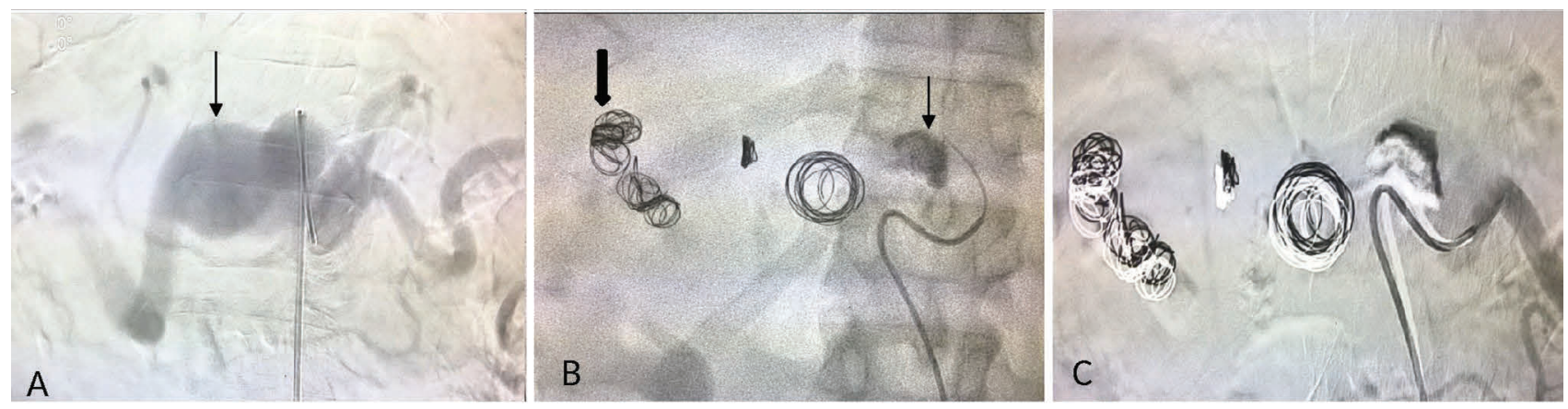

Fig. 2 Celiac axis angiogram showing a large pseudoaneurysm of common hepatic artery (A, arrow) with postembolization angiogram (B, C) showing distal occlusion of aneurysm with coils (B, thick arrow) and glue Lipiodol plug (B, thin arrow) at the mouth of the aneurysm and no residual aneurysm filling $(\mathbf{C})$.

or malignancy, and manipulation of the biliary tract. The noniatrogenic causes encompass trauma, acute and chronic pancreatitis, arteriosclerosis, polyarteritis nodosa (PAN), necrotizing vasculitis, infection, and hepatocellular carcinoma, all of which were ruled out in our patient. ${ }^{3}$ However, as seen in our patient, chronic dissection of the common hepatic artery leading to arterioduodenal fistula presenting as melena is very unusual and not reported previously. ${ }^{4}$ In cases of dissecting visceral artery aneurysms, the most common treatment modality offered was surgery, but with immediate mortality approaching $5 \%$. In our patient, early diagnosis and nonsurgical, endovascular management proved beneficial with excellent outcomes in the short term, without need for surgical management. $^{5}$

\section{Conclusion}

We present the case of a 54-year-old man, a chronic alcoholic who presented to the emergency department with intermittent melena, in whom common hepatic artery dissecting pseudoaneurysm leading to arterioduodenal fistula was found to be the cause, managed immediately with interventional radiology techniques. In cases of melena that are difficult to diagnose through traditional means, early imaging and interventional management can prove beneficial and improve short-term survival.

\section{Funding \\ None.}

\section{Conflict of Interest}

None.

\section{References}

1 Gondolesi GE, Matsumoto C, Wayne M, Schwartz ME. Post-traumatic pseudoaneurysm of the common hepatic artery with duodenal fistula. HPB (Oxford) 2002;4(4):183-186

2 Beerle C, Soll C, Breitenstein S, Grieder F. Spontaneous rupture of an intrahepatic aneurysm of the right hepatic artery caused by segmental arterial mediolysis. BMJ Case Rep 2016;2016:18 [PII: bcr2015214109]

3 Luckhurst CM, Perez C, Collinsworth AL, Trevino JG. Atypical presentation of a hepatic artery pseudoaneurysm: a case report and review of the literature. World J Hepatol 2016;8(18):779-784

4 Higashiyama H, Ishii M, Fujimoto K, et al. Dissecting aneurysm of the hepatic artery caused by an isolated spontaneous celiac trunk dissection. Ann Vasc Surg 2014;28(5):1316. e7-1316.e13

5 Wang HC, Chen JH, Hsiao CC, Jeng CM, Chen WL. Spontaneous dissection of the celiac artery: a case report and literature review. Am J Emerg Med 2013;31(6):1000.e3-1000.e5 